EPSC Abstracts

Vol. 15, EPSC2021-473, 2021

European Planetary Science Congress 2021

(C) Author(s) 2021. This work is distributed under

the Creative Commons Attribution 4.0 License.

\title{
Wheel-regolith interactions on small-body surfaces
}

\author{
Cecily Sunday ${ }^{1,2}$, Naomi Murdoch ${ }^{1}$, Gerald Imhof ${ }^{1}$, Simon Tardivel ${ }^{3}$, Stephan Ulamec ${ }^{4}$, and Patrick \\ Michel $^{2}$ \\ ${ }^{1}$ Institut Supérieur de I'Aéronautique et de I'Espace, Department of Electronics, Optronics, and Signal Processing (DEOS), \\ toulouse, France (cecily.sunday@isae-supaero.fr) \\ ${ }^{2}$ Université Côte d'Azur, Observatoire de la Côte d'Azur, Centre National de la Recherche Scientifique (CNRS), Laboratoire \\ Lagrange, Nice, France \\ ${ }^{3}$ Centre National d'Études Spatiales (CNES), Toulouse, France \\ ${ }^{4}$ German Aerospace Center (DLR), Cologne, Germany
}

\begin{abstract}
We conduct experiments using a single-wheel testbed and simulations using the Soft-Sphere Discrete Element Method to study wheel-regolith interactions on small-body surfaces. We analyze wheel sinkage and traction on different surface materials and we discuss the influence that lowgravity has on rover maneuverability.
\end{abstract}

\section{Introduction}

Wheeled locomotion systems have been successfully operated on the surfaces of the Moon and Mars for decades. Though wheeled rovers have been proposed for small-body exploration [1], they have yet to be tested on the surface of a small moon or asteroid. Driving on a granular surface in a milli or micro-gravity environment will be challenging, but possible. In fact, a rover will be deployed to the surface of Phobos as part of the upcoming Martian Moons exploration mission [2]. The MMX rover will not only demonstrate the feasibility of driving in low-gravity, but it will greatly improve our understanding of regolith dynamics on small body surfaces.

In addition to the navigation cameras, the MMX rover includes two additional cameras, known as the WheelCams. These cameras will capture the direct interaction between the rover's wheels and the surface of Phobos [2]. The WheelCam images will be used to determine surface properties like grain size distribution and shape, friction angle, cohesion, and bearing strength. In anticipation of the MMX mission, we are using experimental and numerical methods to develop techniques to analyze and interpret the WheelCam images. In fulfillment of this objective, we are conducting single-wheel driving tests on different surface materials and are studying the influence that friction, cohesion, and gravity have on the flow behavior of the regolith and the driving performance of the rover.

\section{Experiments}

The WheelCam testbed at ISAE-Supaéro (Fig. 1) [3] is being used to analyze wheel and regolith behavior from the perspective of the MMX rover WheelCams. In this set-up, a motor is used to rotate a 3D printed wheel at a constant speed, and a set of horizontal and vertical sliders allow the wheel to move freely in the horizontal and vertical directions. Various sensors are used to measure the sink and slip of the wheel, and two cameras are positioned to capture the wheel-regolith 
interaction from the same perspective as the MMX WheelCams. Tests are conducted in different granular materials in order to catalogue the resulting trench forms and sinkage levels, and to capture any observable disturbances caused by the wheel's grousers.

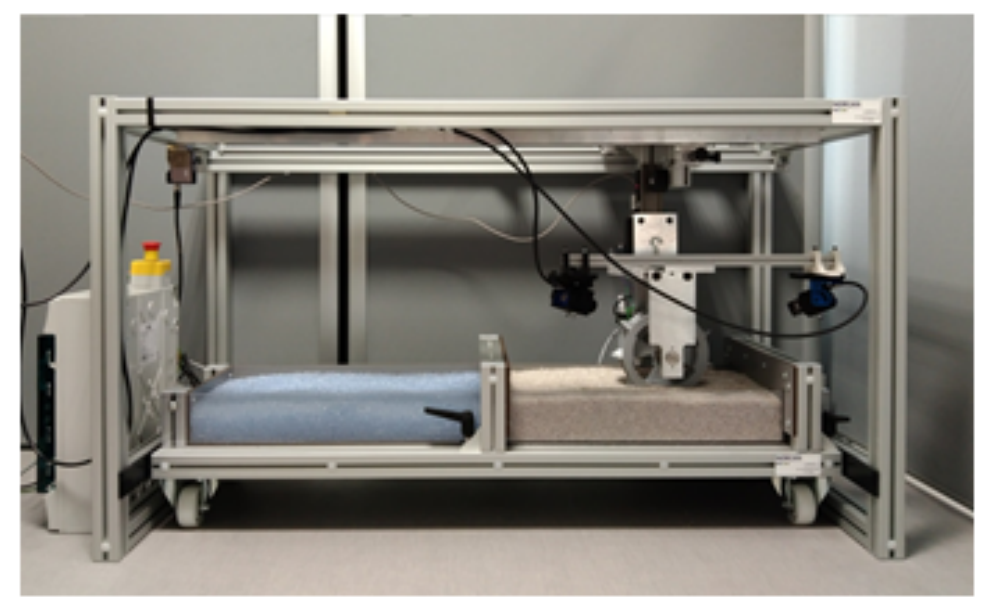

Fig. 1: The ISAE-Supaéro WheelCam testbed [3]

\section{Simulations}

In addition to conducting experiments, we also simulate the rover wheel traversing a bed of granular material using the Soft-Sphere Discrete Element Method (SSDEM) and the open-source code Chrono $[4,5]$. The wheel geometry is based on a simplified version of the MMX rover wheel design [6], and the granular bed consists of approximately 200,000 spherical particles which are 5.5-6.5 mm in diameter (Fig. 2). In the simulations, the wheel sinks into the bed under gravity and then rotates at a constant and controlled velocity. The wheel's behavior is evaluated using metrics like sinkage and slip, and its performance is compared for different types of surface materials (i.e., different friction and cohesion levels) and different gravity levels.

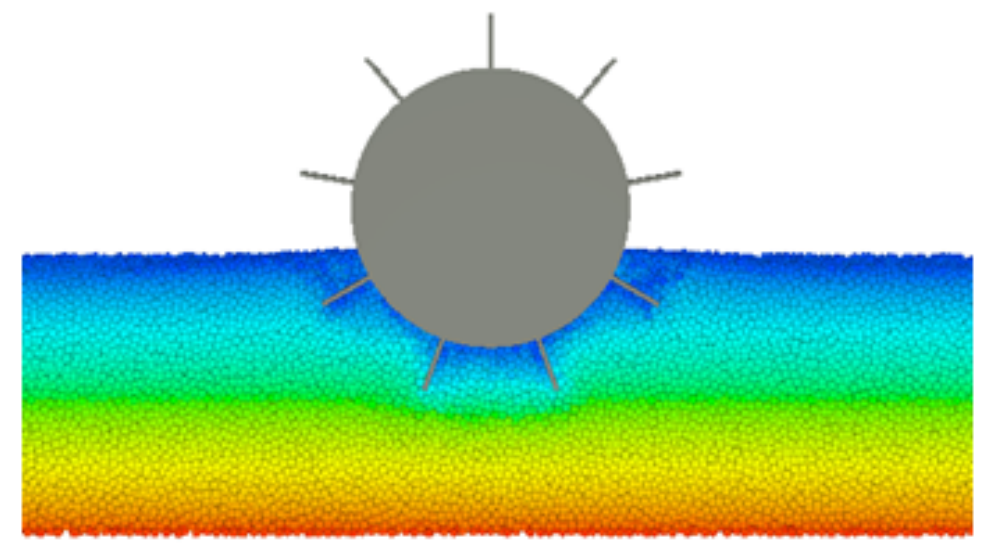

Fig. 2: Simulated rover wheel sinking into a bed of cohesion-less spherical beads under Phobos gravity. The particles are colored by initial distance from the surface of the bed.

\section{Results}

By combining the experimental and numerical results, we can analyze wheel-regolith interactions for a wide range of material cases. We see that wheel sinkage varies drastically according to the friction angle and the cohesion of the surface material. However, the sinkage remains constant across 
different gravity levels. This observation is consistent with findings from parabolic flight experiments [7]. The numerical simulations allow us to study the flow of the regolith directly under the rover wheel in more detail. Recent impact experiments have shown that granular material becomes fluidized more quickly under reduced gravity conditions [8]. Here, we look for the transition from the quasi-static to the inertial flow regime when the rover starts moving to see if fluidization of the material in low-gravity restricts the maneuverability of the rover.

\section{Acknowledgements}

We would like to thank ISAE-Supaéro and the Centre National d'Études Spatiales (CNES) for financially supporting this research effort. The simulations were conducted using HPC resources from CALMIP under grant allocation 2019-P19030.

\section{References}

[1] Wilcox, B. and Ross M. J. "The MUSES-CN nanorover mission and related technology." IEEE Aerospace Conference. Proceedings (Cat. No. 00TH8484). Vol. 7. IEEE, 2000.

[2] Michel, P., et al. "The MMX rover: performing in-situ surface investigations on Phobos." Earth, Planets, and Space. Accepted. 2021.

[3] Passoni, L., et al. "A Single-Wheel Testbed for Regolith Science Studies." Lunar and Planetary Science Conference. No. 2548. 2021.

[4] Sunday, C., et al. "Validating N-body code CHRONO for granular DEM simulations in reducedgravity environments." Monthly Notices of the Royal Astronomical Society 498.1. 2020.

[5] Tasora, A., et al. "Chrono: An open source multi-physics dynamics engine." International Conference on High Performance Computing in Science and Engineering, Springer, Cham , 2015.

[6] Sedlmayr, H., et al. "MMX-Development of a Rover Locomotion System for Phobos." IEEE Aerospace Conference. IEEE, 2020.

[7] Wong, Y., et al. "Predicting the performances of rigid rover wheels on extraterrestrial surfaces based on test results obtained on earth." Journal of Terramechanics 49.1. 2012.

[8] Murdoch, N., et al. "Low-velocity impacts into granular material: application to small-body landing." Monthly Notices of the Royal Astronomical Society 503.3. 2021. 